\title{
A multi-affinity model for logistics network inspired by bio-system
}

\author{
Zhi-Hua Hu \\ School of economics and management, Tongji University \\ Shanghai, 200092, China \\ Logistics Research Center, Shanghai Maritime University \\ Shanghai, 200135, China \\ Email: zhhu@shmtu.edu.cn \\ Zhao-Han Sheng \\ School of economics and management, Tongji University \\ Shanghai, 200092, China
}

Received 14 February 2012

Accepted 29 August 2013

\begin{abstract}
How can we deal with the increasing scale of large logistics network for the booming economy? The structure, mechanisms and principles upon the logistics network show an evolutionary tendency which is complex, and interesting for designing and optimizing logistics network. The adaptability, stability and evolution capability are attractive for artificial intelligent systems. Inspired by biological systems, the metaphors for the structure and eight primary mechanisms are extracted to design a multi-affinity model for bio-inspired logistics network. Then, by mapping the biological elements and functions to logistics network, the evolutionary structure and simulation approach for logistics network are proposed and investigated by experiments. It is a novel way to study the complexity of logistics network and it is an interesting bionic approach for artificial network model. The research result can be employed to study the emergency features and collective behavior of large-scale logistics network.
\end{abstract}

Keywords: bio-system, biological network, logistics network, bio-inspired model, multi-affinity model

\section{Introduction}

In China, according to the statistic data from the government, there are more than 16000 companies that provide logistics services. Because there are many individual service entities social logistics resources which provide basic warehouses and transport services, the number of logistics companies may be much larger than 16000 . China is so wider in terra that the logistics network is big and complex. Moreover, because of the differences between north and south, east and west regions, the unbalance degree is very high. Several questions are raised in such a situation in China. First, how can we assess the logistics? Second, how can we model the big and complex logistics network? Third, how can we provide tools for regional logistics network planning and optimization? The researchers, policy makers and governors are facing such questions in promoting the researches and development of China's logistics industry.

Therefore, in this study, the author tries to propose an approach for modeling and simulating the evolution and stability of large-scale logistics network. Other than operational scheduling and management, our study tries to propose a general framework that can be employed to study the evolution and stability of logistics network. In literature, this objective is seldom identified. Multi-agent system (MAS), complex network, and graph model are commonly employed to study the network features of logistics or supply chain. However, MAS focuses on the interaction between agents; complex network studies the general statistic features 
upon network; and graph model primarily deals with the connectivity. Based on the previous studies in bio-inspired computational algorithms and systems, an affinity model inspired by bio-system is proposed for logistics network.

Affinity is a concept widely used in biology, bio-chemistry and many branches of bio-systems. The affinity in bio-chemistry is described by the dissociation constant to measure the relationship between a ligand (L) (such as a drug) and a protein (P), i.e. how tightly a ligand binds to a particular protein. Ligand-protein affinities are influenced by non-covalent intermolecular interactions between two molecules such as hydrogen bonding, electrostatic interactions and hydrophobic forces. They can also be affected by high concentrations of other macromolecules, which cause macromolecular crowding. In immune system, affinity is a popular concept. Affinity of antibody to antigen increases during an immune response. The increase is achieved by mutating genes encoded for antibody and subsequent selection of best binder. The immune response process takes place in a special microenvironment, namely the germinal centre. The measures based on affinity can be defined between various types of immune molecules, cells and organs. The measures are used to represent the complex relationships between immune entities. Immune principles action according to affinities and reversely adjust the affinities to express concrete immune functions. In cytokine network that dominates the body functions, affinity is a measure for ubiquitous activation and prohibition relationships. Therefore, it is concluded that the affinity in bio-system defines general relationships between various entities. Moreover, the measures and principles express the biological functions according to affinity and adjust the bio-system by adjusting affinity.

Based on affinity, the feasibility, the modeling skills and the implementation issues will be identified for logistics network modeling and simulation. The following points are made in the study as the contributions. First, logistics network is modeled and analyzed based on bio-system inspired principles. Second, the models and principles are extracted from the mechanisms based on affinity for researches on the evolution and stability of logistics network. Third, affinity-based logistics network model is built and the corresponding functional mechanisms are designed to simulate the evolution and stability of logistics network.
The rest sections are organized as follows. In Section 2, the background of logistics network, bio-inspired logistics network and the pervious studies are investigated. Then, in Section 3, three feasible solutions are discussed. Multi-affinity model is chosen as the preferred solution and studied in Section 4. In Section 5, a simulation platform and some experiments are designed to study the evolution and stability of logistics network. Finally, the study is concluded with future research directions.

\section{Related studied}

\subsection{Designing and simulating logistics network}

Logistics network is a common term that represents system of warehouses and transport channels in regional or national wide. In a logistics network, nodes in logistics chains are dependent; nodes represent warehouses and other facilities that commonly have relatively fixed locations; node can be multi-models so that it can have different functions; channels between nodes can be the flows of transport means, material, capital, human and value; the transforms in time, space and composite dimensions should be supported in the structures of nodes and arcs.

Therefore, several research points can be found by analyzing the above features in literature. First, the models of logistics network, especially multi-model or international transport network, are researched $[8,26]$. Second, location, layout and other design issues are focused on by many scholars [17]. Third, transport problems are investigated, and many scheduling models and tools are proposed, designed and implemented. Moreover, the problems upon logistics network are mostly NP problems, which become the benchmarks for optimization, such as TSP (travel salesmen problem) [18] and VRP (vehicle routing problem) [2]. The fourth type of researches deals with information systems that integrate the resources of warehouses and transportation to serve managerial objectives of logistics system.

In the aspect of structural complexity of the logistics network, two primary categories can be classified: hierarchical (or, chain, layered, multi-echelon) structure, and graph structure. Hierarchical logistics network models the logistical processes by chains of nodes and channels by sequential passing orders, usually of material. In each hierarchy, usually multiple choices can 
be supported, and the connectivity is built between the adjacent hierarchies. Hierarchical structure is simplified and practical for industries and research purposes. Commonly, integer programming model can be built for hierarchical structure [19]. The second type of structure releases the presumption that the connection is restricted between layers. The connections of this type can exist between any two nodes in logistics network $[15,25]$. When the modes of the nodes and arcs are considered, the extended graph models will be used. Therefore, this type of structure is based on Graph. Graph model is suitable for location and routing problems upon logistics network. However, graph model usually increases computation complexity for optimization or simulation.

Designing logistics network is to determine the nodes and arcs corresponding to the distribution of storages and operational entities, and the transport channels. Most literature on logistics network tackle certain design problems [7, 21]. Simulation methods are usually employed to study the capacity or capability of nodes and channels, and stochastic and uncertain features $[23,27]$.

\subsection{Bio-inspired approaches}

Bio-inspired models, algorithms and systems have achieved a lot of results in many research fields [4-6, 22] . Bio-inspired approach has become a key methodology to deal with complex problems [22] . Bio-inspired methodologies have intuitive concepts, models and principles to define relationships and interactive principles among entities in complex systems. Commonly, the evolutionary and emergency capabilities can be revealed by designing mechanisms and simulations. When practical applications have been connected with bio-systems, the metaphors can be utilized to design artificial structures and behaviors. There are many astonishing attempts to analyze and optimize complex systems, and proclaim hidden facets in literature. The most famous bio-inspired algorithms include genetic algorithm [1], neural network [14] , immune system [24]. Multi-agent system simulates society and human decision behavior $[16,20]$. In the above approaches, bio-inspired structures and principles are designed by metaphors from genetic system, neural system, immune system and psychology. Genetic algorithm is usually designed for optimization. However, evolutionary systems based on the bio-inspired structures and principles are also studied.
Neural network is applied in many fields, especially function approximation. Immune algorithm is believed to be superior to traditional evolutionary algorithm. Immune system is novel scheme for artificial intelligent system, which is specially applied widely in security system and evolutionary system. Multi-agent system is a more general system to model the interaction between entities in complex system.

Logistics network is special kind of complex network, whereas there are seldom seen specific bio-inspired models and systems designed for it.

In our previous studies, we have published some research papers on bio-inspired algorithms and computational systems. In [3], an antibody network is proposed to model an architecture of distributed object computation. Some principles are designed for managing distributed object containers inspired by human immune system. In [9], immune principles are employed to design co-evolutionary algorithm with interactive procedure to optimize and evolve the library of design solutions for garment computer-aided system. In [13] , another complex algorithm is proposed to search the districting solutions for large-scale distribution. In [10], a multi-affinity is proposed to model the complex structural relationship among different element set in multi-objective optimization. In [12] , interaction principles in distributed object containers are implemented by immune-inspired principles. A prefetching algorithm is designed according to the bio-inspired structure and logics.

The above researches focus on the abstraction and application of immune-inspired frameworks, models and algorithms. The authors have accumulated much knowledge of bio-inspired approaches and applications. All these previous researches will contribute to this work.

\section{Feasible approaches}

\subsection{Graph-based model}

For all structural patterns for logistics network, graph model may be an ultimate foundation. Hierarchical model is a simplified graph. Other network modeling skills, such as MAS, bio-network, complex network, commonly take graph as prototypes or basic structures. Therefore, in this aspect, graph model is a structural base for logistics network. However, if the logistics network is based on graph model, it should be extended 
to support the modeling features and principles of logistics network.

\subsection{Multi-agent model}

MAS is more flexible for modeling complex problems than graph model. Relationship between agents is modeled by interaction. Moreover, it is a more universal connection than what is provided by graph model. The connections between nodes of network based on MAS are weaker than those in graph. However, the capabilities and behaviors of nodes can be exaggerated in MAS. Moreover, it is advantageous to model dynamics of nodes and arcs by MAS. However, when logistics network is modeled by MAS, although the features and behaviors of the nodes (warehouse or other logistical operational entities) can be implemented in a flexible way, it is difficult to model the transport relationships between nodes in direct and intuitive ways. Furthermore, multi-mode transport and the various flows between nodes connect the relatively static nodes to be a network. Moreover, these flows are commonly homogeneous to any pair of nodes so that it is not so necessary to utilize the full capabilities of agents.

However, although this work does not choose MAS as the direct modeling approach for logistics network, it is a base of the bio-inspired approach discussed in the next section, Section 3.3.

\subsection{Bio-inspired approach}

It is somewhat a philosophy problem to explain the superiority of bio-inspired approaches. However, bio-inspired structures and principles do provide novel solutions for many complex systems and problems in literature. The bio-inspired approaches commonly utilize the structures based on graph and MAS. Bio-inspired approach creates a way to incorporate foundational structure and new principles by metaphors from bio-systems. By analyzing the characteristics of logistics network and the identified issues, it is believed that bio-inspired approach can serve the research in the following points.

First, by utilizing graph model, nodes and arcs are modeled by novel explanation in the context of logistics network. Node represents entity in logistics network, whereas arc represents transport channel or flow between entities in logistics network.

Second, node acquires the capability of agent. However, the properties and behaviors inspired by bio-system for entity can be defined for nodes in logistics network. In bio-system, node can be cell, organ, or other functional entity. The general form of them is employed to represent the entity in logistics network. Entity has an entire lifecycle and it can be controlled by others or control others. As another important fact entity is also a node in flow.

Third, the arcs here are multi-mode. Between any two nodes, there are probably more than one connection to represent parallel channels and flows. Flows may be driven by the forces from nodes. However, the passing capacity will determine the flow status. The flow also will only match the limited set of nodes. In other words, the category or features of node will determine the flow or channel connected with this node. These situations from logistics system are similar with signal channels in bio-systems.

Fourth, there are rules that dominate logistics network just like the principles that express the biological functions. By reconstructing the metaphors, logistics network can acquire the features of bio-systems, which reversely produce the emergent and evolutionary features in bio-systems. The typical features of bio-systems are self-stable, self-adaptive, self-protective, self-learning, evolutionary and emergent, which are beneficial to the globally optimized logistics network. In this work, the evolution and stability of logistics network are focused on.

\section{Multi-affinity model}

\subsection{Inspirations from bio-system}

\subsubsection{Multi-affinity}

(1) Neural network system

In neural network system, biological neurons are connected with each other. In general, a biological neural network is composed of a group or groups of chemically connected or functionally associated neurons. A single neuron may be connected to many other neurons and the total number of neurons and connections in a network may be extensive. Connections that are called synapses are usually formed from axons to dendrites, though dendrodendritic microcircuits and other connections are possible. Apart from electrical signaling, there are other forms of signaling that arise from neurotransmitter diffusion. These forms of signaling have an effect on electrical 
signaling. The tremendous capabilities of response and computation of neural network are implemented by these connections of different types, which build the relationships among neurons. In this study, this connectional capability for computation is presented by affinity. Because the connections between neurons have different characteristics, they are presented by multi-affinities. By representing these connections by affinity measures, the functions of neural network are modeled in a new way for logistics network in this work.

(2) Endocrine system

In physiology, the endocrine system is a system of glands. Gland secretes a type of hormone into bloodstream to regulate the body. The endocrine system is a signal system like neural system. Hormones regulate the functions of an organism, including mood, growth and development, tissue function, and metabolism. Endocrine system is made up of a series of ductless glands that produce chemicals called hormones. Typical endocrine glands are the pituitary, thyroid, and adrenal glands. Features of endocrine glands are, in general, their ductless nature, their vascularity, and usually the presence of intracellular vacuoles or granules storing their hormones. In contrast, exocrine glands, such as salivary glands, sweat glands, and glands within the gastrointestinal tract, tend to be much less vascular and have ducts or a hollow lumen. In endocrine system, glands, hormone and signals construct the elements. Glands secret hormones that are connected by signals. Although we can consider more complex structures of endocrine system, the relationships indicated above can be abstracted by affinity measures between endocrine entities. Then, the functions expressed by endocrine system can be deduced by the affinity of related entities. Various types of signals in endocrine system can be modeled by multi-affinities. These relationships connect different glands and hormones to express endocrine functions.

(3) Immune system

An immune system is a system of biological structures and processes within an organism that protects against diseases by identifying and killing pathogens and tumor cells. It detects a wide variety of antigens, from viruses to parasitic worms. It distinguishes them from the organism's own healthy cells and tissues. Detection is complicated because pathogens can evolve rapidly by producing adaptations that avoid the immune system and allow the pathogens to successfully infect their hosts. To survive from this challenge, even simple unicellular organisms such as bacteria possess enzyme systems that protect against viral infections. The typical vertebrate immune system consists of many types of proteins, cells, organs, and tissues that interact in an elaborate and dynamic network. As a part of these complex immune responses, the human immune system adapts over time to recognize specific pathogens efficiently. This adaptation process is referred to as "adaptive immunity" or "acquired immunity". This process creates immunological memory that is created from a primary response to a specific pathogen. Immunological memory provides an enhanced response to secondary encounters with those same and specific pathogens. This process of acquired immunity is the basis of vaccination. After the body gains immunity towards a certain pathogen, when infection by that pathogen occurs again, the immune response is called the secondary response.

Immune system expresses the following functions: self and non-self discrimination (SNSD), danger detection and body protection, and stability maintenance. These functions are attractive to artificial systems. However, how can the immune system obtain such functions? The innate structures and principles have inspired many scholars to design novel computational architectures and models. Antibodies and other immune cells or entities cooperate to detect and destroy antigens or pathogens by the foundational structure of immune network, where affinity is a universal measure for the entities including genotypes of phenotypes. Immune networks, especially cytokine network, express immune functions by affinities between entities in the network. The entities contain antibodies, antigens and various forward and feedback signals. Affinity is a concept widely used in immune theory, where multi-affinity is employed to represent the complex relationships among immune entities.

(4) Other bio-systems

Like genetic system, eco-systems are generally studied as populations and networks upon population. Biological entities express their specific biological functions by cooperation through network. In genetic system, the evolution is achieved by evolutionary operations upon chromosome population. The genetic operations generate children chromosomes from 
parents. These relationships can be modeled as a dynamic network. The connections discriminated by evolutionary operators can be modeled as multi-affinities to represent the evolutionary pressure and capability.

Eco-system is balanced and stabilized by food chains Eco-system is a universal network to coordinate various populations. The flows, foods, controls and feedbacks, are all streamed in eco-network to achieve ecological functions. These coordinative flows can also be modeled as multi-affinities which then become the foundations for ecological regulations.

In a summary, all bio-systems show features of evolution and stability, which attract the development of artificial systems by extracting underlying structures and functional principles in bio-systems. Affinity can be employed to abstract the coordinative or competitive signals or relationships in networks of bio-entities. Multi-affinity model can be employed to represent the flexible and strong coordinative relationships in bio-systems.

\subsubsection{Evolutionary mechanisms}

In the following, various biological chromosomes, cells, organs or other entities in bio-systems are uniformly titled as bio-entity. Bio-entities connect with each other by biological network where affinities measure the relationships in the network. Moreover, biological principles express the biological functions based on the multi-affinity measures on the network. As mentioned in Section 4.1.1, bio-systems show attractive features that inspire the design of artificial systems. Evolution is one of the most important features, which is helpful to design logistics network. In the following, evolutionary mechanisms are studied in three categories including bio-entity, biological connection, and biological network.

\section{(1) Bio-entity}

Bio-entities can be self or non-self to the biological body. Self and non-self entities commonly have the corresponding or complementary structures and functions. Therefore, the first mechanism is to discriminate the self and non-self by structure analysis and function matching. The second is about lifecycle management. As for the processes of creating and eliminating bio-entity, and the invasion event, state diagram can be employed to describe the status and the events that trigger the transfers of states. The second classification scheme is the scale of individuals: individual, group or population. The above two mechanisms are designed for individual bio-entity. Third, the group and population mechanisms primarily include construction and elimination of groups, in other words, group lifecycle management. Fourth, another important mechanism deals with the member entrance and existence of bio-entities, namely membership management. Fifth, affinity measures can be defined between the following individuals, populations and their corresponding genotypes: bio-entity, bio-entity group and bio-entity population. The quality of individuals and population evolve by the above mechanisms first and then depend on the following ones.

(2) Biological connection

Biological connections are implemented in bio-systems primarily by physical connections and signals. It is presumed that any connection can only connect two individuals, or one bio-entity connects with itself. The first mechanism is about how the connection can be built upon two bio-entities and what about the matching conditions. This type of connection is primarily determined by the structural principles of the system. Therefore, it is named as structural connection. The type of connection can be measured by the structural affinity between two bio-entities. Second, the connection can prohibit and strengthen the specific status of connected bio-entities by the comparative values of affinity of the connection to the bio-entities. This mechanism builds the relationship between bio-entities and connections. This type of mechanism is called as entity-connection coordination. Third, what the connection loads should pass through the bio-entities. Therefore, the prohibition and the produced effects should be measured on the bio-entity by the flow. It can be named as entity passing capability. All these mechanisms take affinity as the measures to assess the coordination or propitiation relationships among the composite set of bio-entities and connections.

\section{(3) Biological network}

The concepts of biological network can be classified into three categories in different levels: nature, social and artificial levels. Food chain is a typical type of nature biological network. Social network theory attracts more and more people to devote to the study. Artificial networks inspired by biological systems have been developed in many areas. In computational intelligence, neural network, immune network and 
bio-network are typical representations. It is believed that the bio-inspired mechanisms are beneficial to design artificial systems.

The mechanisms on biological network take charge of the control of the biological system in a higher and more general way than ones of bio-entity and biological connection. First, the ownership management mechanism controls the lifecycle of the bio-entities and connections, and groups of them. These functions indeed are natively supported by the entities themselves. Second, the coordination mechanisms design the rules among entities commonly to deal with specific objectives. These rules are usually inspired by biological systems. Coordination is believed to be general principle in societies of creatures. Moreover, among these mechanisms, feedback is an important one to achieve the survival capability of the network. Third, the evolution mechanisms are principles inspired by bio-systems when they are taken as a system of population. Evolutionary principles propel the development of bio-system and build the order for the population by unintuitive ways.

These mechanisms are all iterated in the context of evolutionary mechanisms because evolution is believed to be the most general principle and to be the objectives of other mechanisms.

\subsection{Model formulation}

Corresponding to the studies above, three parts are considered in the formulation: structural elements, coordination mechanisms and evolutionary principles. Because the boundary between the last two is not very clear, they are merged into principles. Moreover, because the studies on the model are investigated for logistics network, the extensions are provided to map biological system to logistics system.

\subsubsection{Structural model}

In the context of bio-system, the basic elements of the model include bio-entities, connections, and invaders. These elements construct a biological network. The network is represented by Eq. (1), where three sets are defined and the network is defined upon them.

$$
\begin{aligned}
& N E T=(N O D E, C O N N, I N V D) \\
& N O D E=\{1,2, \cdots, N N\} \\
& C O N N=\{1,2, \cdots, N C\} \subseteq N O D E \times N O D E \\
& I N V D \subseteq N O D E
\end{aligned}
$$

Because of introducing $I N V D$, the model is different from traditional graph model. Here, the invaders are part of bio-entities because it is presumed that the invaders attack the network by attacking the bio-entities in the network. Based on these definitions, the properties of the entities, connections and invaders are defined.

The properties of entities have two types. The first is native properties and the second is connection properties. A vector $P N O D E=\left(P_{1}, P_{2}, \cdots\right)$ is defined to represent the properties on the entity node $\in N O D E$, as computed in Eq. (2) for each property. It is presumed that $P_{i}$ (node) is independent with other entities and connections. Therefore, its value is self-determined. For these reasons, there may be properties that can be determined by other entities or connections. However, the relationships among entities can be believed that they are determined by corresponding connections. Therefore, only the properties of entity to connection are considered in the following.

$$
\begin{aligned}
& P N O D E=\left(P_{1}, P_{2}, \cdots\right) \\
& P N O D E(\text { node })=\left(P_{1}(\text { node }), P_{2}(\text { node }), \cdots\right), \\
& \text { node } \in N O D E
\end{aligned}
$$

The connections model the relationships between entities and connections, or entities and other entities. Moreover, in the structural model, only the basic structures and mechanisms are defined so that only the relationship between a single entity and a single connection is considered.

$$
\begin{aligned}
& P N O D E C O N N=\left(P_{1}, P_{2}, \cdots\right) \\
& \text { node } \in N O D E, \text { conn } \in \text { CONN }: \\
& P N O D E C O N N(\text { node }, \text { conn })= \\
& \left(P_{1}(\text { node }, \text { conn }), P_{2}(\text { node }, \text { conn }), \cdots\right)
\end{aligned}
$$

Similarly, the property vector on connection can be defined in Eq. (4).

$$
\begin{aligned}
& P C O N N=\left(P_{1}, P_{2}, \cdots\right) \\
& P C O N N(\text { conn })=\left(P_{1}(\text { conn }), P_{2}(\text { conn }), \cdots\right), \\
& \text { conn } \in \text { CONN }
\end{aligned}
$$

In the above definitions, the property itself is not assigned yet. Because the purpose of this study is to build a multi-affinity model for logistics network, the properties should support the operations and management of logistics network.

For the invaders, the properties can be classified into two types. The first is native properties and the second is relationship measures between invaders and entities. The native property vector is defined similarly as Eq. (2) and Eq. (5). Because it is defined in Eq. (1) that the 
invader is in the set of entities, invaders have the properties defined on entities.

$$
\begin{aligned}
& P I N V D=\left(P_{1}, P_{2}, \cdots\right) \\
& P I N V D(\text { inv })=\left(P_{1}(\text { invd }), P_{2}(\text { invd }), \cdots\right), \\
& \text { invd } \in I N V D
\end{aligned}
$$

Because an entity can be a biological entity or an invader, a predication is defined in Eq. (6). The enumeration set is defined to represent the type of entities, Invader or Entity. Then the formula is defined to assign the type of an entity.

$$
\begin{aligned}
& \text { EORI }(\text { node }) \in\{E, I\}, \text { node } \in N O D E \\
& \text { EORI }(\text { node })=\left\{\begin{array}{l}
I, \text { if }(\text { node } \in I N V D) \\
E, \text { if }(\text { node } \in N O D E \backslash I N V D)
\end{array}\right.
\end{aligned}
$$

\subsubsection{Multi-affinity measures}

Before the principles on network are studied, some affinity measures are proposed in advance. Affinity is employed to measure the relationship among the elements of the network. There are multiple measures to be defined, because various relationships are considered to cover the possible combinations. In this study and based on the previous studies, the affinity measures share the similar formation as Eq. (7). The parameters of the affinity measures can be single entity (biological entity, connection and invader) or entity set of these entities.

$$
\begin{aligned}
& \text { Aff }\left(\text { ele }_{a}, \text { ele }_{b}\right) \\
& =\operatorname{aff}\left(P\left(\text { ele }_{a}\right), P\left(\text { ele }_{b}\right), P\left(\text { ele }_{a}, \text { ele }_{b}\right)\right)
\end{aligned}
$$

In the previous studies $[10,11]$, genotypes and

\begin{tabular}{|c|c|c|}
\hline Notation & Candidates & Comment \\
\hline$n$ & node $\in N O D E$ & A single entity \\
\hline$N$ & $N O D E S \subseteq N O D E$ & Entity set \\
\hline$c$ & conn $\in C O N N$ & A single connection \\
\hline C & $C O N N S \subseteq N O D E$ & Connection set \\
\hline$i$ & invd $\in I N V D$ & A single invader \\
\hline$I$ & $I N V D S \subseteq I N V D$ & Invader set \\
\hline NET & $N E T$ & The entire network \\
\hline
\end{tabular}
phenotypes are discriminated to show the specific relationships about the belonging relations. In Eq. (7), these features are also supported by setting the parameter to be a general set of specific type of entities. The candidates of the parameters are shown in Table 1.

Table 1 . The candidates of affinity parameters

Therefore, 49 affinity measures can be defined. In the specific context of applications, not all affinity measures are valuable to be utilized. In this study of logistics network, the following affinity measures are representative: $A f f_{n-N}, A f f_{n-n}, A f f_{c-C}, A f f_{c-c}, A f f_{i-n}$ and $\operatorname{Aff}_{n-c}$.

\subsubsection{Principles}

As studied above, the principles can be mechanisms of coordination (including competition), and evolutionary principles. In the following, by analogy of biological systems and the design purpose of logistics network, some principles are designed.

(1) Invasion detection

Self-protect is a direct purpose of biological systems. The attack from the environment of biological entities can be represented by invader entities and its property vectors. Another aspect is the invasion scale deduced by invader entity. Moreover, the attack may be launched by more than one invader. In Eq. (8), an invasion is defined by three elements: invader entity is a kind of entity that attacks the network; the parameter $t w=\left[t_{1}, t_{2}\right]$ is defined to construct a time window, and invd $\in I N V D$, scale $\subseteq N O D E$. The scale is a set of entities as direct invaded targets.

$$
\text { INVASION (invd,tw, scale })
$$

The detection process matches the invader by a leading entity. In Eq. (9), the matching predication is defined, where invd $\in I N V D$, scale $\subseteq N O D E$, node $\in N O D E$. In practical application, the matching can be a degree. The matching degree is determined by an affinity measure between invader and biological entity, as defined in Eq. (10).

$$
\begin{gathered}
\operatorname{MATCH}(\operatorname{INVASION}(\text { invd }, \text { tw }, \text { scale }), \text { node }) \in\{0,1\} \\
\operatorname{Aff}_{i-n} \rightarrow \text { MATCH }
\end{gathered}
$$

It is not necessary to claim that the matched entity is singular. However, it is presumed that the matched entity exists anyway.

$$
\mid\{\text { node } \mid \text { MATCH }(\operatorname{INVASION}(\text { invd, tw }, \text { scale }), \text { node })=1\} \mid \geq 1
$$

In the following, INVASION is employed to represent the set of invasions. Therefore, invs $\in$ INVASION is used to represent Eq. (8). For Eq. (11), another notation is introduced to represent the matched entity by a concise way in Eq. (12).

$$
M A T C H(\text { invs } \in I N V A S I O N, N E T) \subseteq N O D E
$$

(2) Invasion response

The network produces responses to the invasion by collective behaviors. First, the target scale of the invasion determines the scale of cooperation to resist it. 
Second, the matched entities will organize the members to construct a local network for response. Third, the energy requirement and acquisition will absorb a set of efficient entities to assist the response. Therefore, the features of invasion response include the following points: first, it is a collective behavior by coordination; second, it involves a set of balanced and efficient entities; third, the response is performed by a form of network.

All these entities for invasion response and coordination are organized into a directional sub-network of the entire network, as defined in Eq. (13). The sub-network induced by an invader to the network primarily relates three affinity measures, as denoted in Eq. (14).

$$
\begin{aligned}
& \text { RESPONSE (invs } \in I N V A S I O N, N E T) \subseteq N E T \\
& \left.\begin{array}{l}
\operatorname{Aff}_{i-N} \\
\operatorname{Aff}_{i_{-N E T}} \\
\operatorname{Aff}_{n-n}
\end{array}\right\} \rightarrow \text { RESPONSE }
\end{aligned}
$$

(3) Bio-entity tolerance

In this study, only the entities that the capacity is matched with the invader can respond to an invader. First, the genotype of the entity will determine whether it can take the type of the invasion. This determination is named as genotype matching. Second, based on the capacity of the entity, it is determined whether it can take the strength of the invasion. This process is named as strength matching. Third, the entity will tolerate the invasion when the acquired energy from response is far less than the expected energy by the entity. It can be profit tolerance. These three types of tolerance will prohibit the activation of response. In this study, it is simplified in Eq. (15), where invs $\in$ INVASION and node $\in N O D E$. In the equation, the value of tolerance is higher when the invasion will be tolerated most. In Eq. (16), three types of tolerance of bio-entity are aggregated. The tolerance relationship can be expressed by the affinity between invader and entity, as in Eq. (17).

$$
\begin{gathered}
\text { TOLERATEN }(\text { invs, node }) \subseteq[0,1] \\
\text { TOLERATEN }(\text { invs, node }) \\
=A G G\left(\begin{array}{l}
\text { GenotypeMatching }(\text { invs, node }), \\
\text { CapacityMatching }(\text { invs, node }), \\
\text { ProfitTolerrance }(\text { invs }, \text { node })
\end{array}\right) \\
A f f_{i-n} \rightarrow \text { TOLERATE }
\end{gathered}
$$

(4) Channel tolerance
In biological networks, the material, control signals, information and inherited knowledge, and many other tangible or intangible, visible or invisible things construct the complex structures of flows. Here, channel is a direct name of connection in network. For each connection, three types of tolerances also can be defined similarly as Eq. (18) and Eq. (19), where invs $\in$ INVASION and conn $\in$ CONN . In the previous section, the affinity between invader and connection is not defined explicitly. In Eq. (20), the tolerance is determined by the affinity between invader and entity, and the affinity between entity and connection. These type affinities are transferred to construct the relationship between invader and connection.

$$
\begin{gathered}
\text { TOLERATEC }(\text { invs, conn }) \subseteq[0,1] \\
\text { TOLERATEC (invs,conn }) \\
=A G G\left(\begin{array}{l}
\text { GenotypeMatching }(\text { invs, conn }), \\
\text { CapacityMatching }(\text { invs, conn }), \\
\text { ProfitTolerrance (invs, conn })
\end{array}\right)
\end{gathered}
$$$$
\text { Aff }_{i-n} \cdot A f f_{n-c} \rightarrow \text { TOLERATE }
$$

The entity and channel tolerance define the entrance of response. However, how does the response be performed? What about the execution process? In the following principles, inspired by biological systems, another four mechanisms are studied.

(5) Idiotype cascading

It is an interesting and reasonable way to response and deal with tasks in biological population by idiotype cascading or increasingly produced hierarchical structure of population. Idiotype is a specific concept in immune system. The antibodies will express their function by a cascading intersected chain or network. First, it is also a collective behavior for the especially large and strengthened tasks. Second, it is a dynamic structure to ensure the adaptive force combination and optimization for the outside tasks. The cascading structure is constructed by requirements and it can be disposed at the end of the task. In the cascading process, the key processes include the following points. First, which will be appended into the response network? Second, which will be removed from the network at a specific time or period?

Therefore, the cascading process determines the affinity between the entity and the cascading sub-network, and between the entity in the sub-network and the sub-network. As discussed above, the appending 
process and removing process are considered in Eq. (21) and Eq. (22). In the two formulas, when the network instance is taken as container of entities, it refers to the entity set of the network. It is obvious that the values are determined by the affinities between entity and sub-network, although the entity belongs to the sub-network or not. In Eq. (23) and Eq. (24), the affinities are given.

$$
\begin{gathered}
\text { net } \subseteq N E T, \text { node } \in N O D E \backslash N E T: \\
A P P E N D(\text { net }, \text { node }) \in[0,1] \\
\text { net } \subseteq N E T, \text { node } \in \text { net }: \\
R E M O V E(\text { net }, \text { node }) \in[0,1] \\
\text { Aff }_{n-N} \rightarrow \text { APPEND } \\
\text { Aff }{ }_{n-N} \rightarrow \text { REMOVE }
\end{gathered}
$$

(6) Proliferation

Proliferation is an interesting mechanism to reproduce productivity when the demand is increased for a lot. It is a general principle in immune system and ecosystem when the invasion happens. In bio-system, in the process of proliferation, entities are chosen and duplicated so that the scale of the superior entities can play an important role in the detection and elimination of invaders. Therefore, in artificial systems, the metaphor of proliferation can be expanded in two ways. First, the entity is duplicated so that the superiors in the population increased. Second, the capacity of the entity is enhanced so that it can deal with the strengthened invaders. In Eq. (25), Eq. (26), Eq. (27) and Eq. (28), two types of proliferation and the affinity determination rules are defined.

$$
\begin{aligned}
& \text { PDUPLI }(\text { node } \in \text { NODE }) \subseteq N O D E \\
& \text { node }= P S T R E N(\text { node } \in \text { NODE }) \\
& A f f_{n-N} \rightarrow \text { PDUPLI } \\
& \text { Aff } f_{n-N} \rightarrow \text { PSTREN }
\end{aligned}
$$

\section{(7) Hypermutation}

Hypermutation is also a concept in immune system. New entity is generated and its structure and properties are altered to produce diversity. Proliferation duplicates new entities by possible minor modification. It is a way for convergence to keep the superior ones in the generation. However, hypermutation is a way to introduce novel ones to the population. In the aspect of energy, hypermutation increases the level of energy. However, it also produces or strengthens the pressure of the network. Therefore, whether it can be performed upon the network is determined by the network's capacity and energy. In Eq. (29) and Eq. (30), the process of hypermutation and the affinity determination rule are provided.

$$
\begin{gathered}
\{\operatorname{HYPERM}()\} \cup N O D E \rightarrow \text { NODE } \\
\text { Aff }{ }_{n-N} \rightarrow \text { HYPERM }
\end{gathered}
$$

(8) Elimination

Proliferation and hypermutation are mechanisms to enlarge bio-entity population. Elimination is designed to decrease the population size. Therefore, these three mechanisms take action together to stabilize the population dynamically. When the processing pressure of the network is increasing, proliferation and hypermutation will take place to increase the capacity of the network. After the peak time, abundant capability should be reduced to keep the population stable. In another aspect, the combination of these mechanisms will optimize the network and continuously constitute an evolutionary process. In Eq. (31) and Eq. (32), although only the entity set is considered, the altering of it will deduce the modifications of connections in the network.

$$
\begin{gathered}
N O D E \backslash\{\text { ELIMI }()\} \rightarrow \text { NODE } \\
A f f_{n-N} \rightarrow \text { HYPERM }
\end{gathered}
$$

These mechanisms are inspired by biological systems. By them, the biological systems show the features of adaptability, stability and evolution.

(1) Adaptability

The adaptability here refers to several points. First, the capability of detecting and responding to the invaders should be adaptive. Second, the interactions of the network with the environments not only produce pressure to the network for energy consumption, but also help the network acquire the energy. Therefore, the balance between the energy production and consumption is a key factor for the adaptability. Third, when the invasion can not be removed, the proliferation of capability should adjust the structure of the network to cater for it.

(2) Stability

Ecosystem and immune system are two typical systems that succeed in maintaining their stability of biological systems. The structures and principles of these systems achieve the objective of survival of the entire biological population. The stability maintenance capability can be detailed in the following two aspects. First, the time to recover from invasion is a direct measure for stability when bio-system faces the 
disturbances produced by invaders. The flexible capability assignment and redeployment affect the system stability. The second concerns about the survivable capability for long term. The ultimate objective is believed to be survival in the history and in the future. Then, the capability to deal with the invaders and other emergency situations determines the achievement of these objectives.

\section{(3) Evolution}

Evolution controls natively the change in the inherited traits of a population of organisms through successive generations. As well as adaptability and stability, evolution is the most attractive feature for artificial intelligent systems. The above mechanisms are evolutionary principles to achieve the evolutionary features. The evolution can be categorized into two aspects, structure evolution and capability evolution. During the evolution processes, the structure of the network will be evolved to a better one to cater for the dynamic environments and invaders. The network capacity should be adaptive and flexible to the survival of the network and the fluctuated attacks from the environments.

\subsection{Extensions for logistics network}

The structural model and mechanisms are designed in the previous sections. How can this model be applied in logistics network? Can all the structural elements and formulas be designed according to the nature of logistics network? Can it be simulated? And, can it be measured? In this subsection, the first two questions will be answered in Table 2. The last two will be dealt with in Section 5.

\section{Simulation}

\subsection{Simulation platform}

The above concepts and models have been employed to design logistics systems. However, the concrete systems involve more sophisticated details that may have weak relationship with the main purpose of this work. Or, the studied features are difficult to be focused on in practical applications. Therefore, in the simulation study, we focus on revealing the evolution and stability of the logistics network inspired by bio-system.

A simulation platform is built upon the following rules or simplified specifications based on the principles and mechanisms studied in Section 4.
1) A logistics network is considered. The nodes have the ability of storage, loading and unloading. The transport means are not discriminated. The transport capacities including travel time and volume are constrained.

2) Logistical demand is defined by weight, a target and a preferred time with penalty for delay.

3) The key properties and principles are redefined in Table 3 .

4) The simulation process is discrete-time triggered. Moreover, for simplicity, at anytime, only one task is generated and running. The simulated logistics network is generated upon a grid of nodes and connections. Then, the simulation procedures are designed as Algorithm 1.

Algorithm 1 (Simulation Procedure)

Input $|N O D E|$ : the size of node set NDPOINTS : the number of candidate demand nodes.

NTPOINTS : the number of target nodes.

Output Runtime data for analysis

Processes

Step 1 Network initialization

Initialize the network structure and property configurations;

Initialize the demand nodes and aim nodes configurations.

Step 2 Demand generation

Generate the demand node and parameter generation;

Conduct node matching.

Step 3 Network reconfiguration by proliferation

Assess the efficiency of the network;

Conduct proliferation to strengthen the capability;

Eliminate the inferiors with fewer operation times.

Step 4 If not terminated Then GOTO Step 2

Step 5 Generate statistical results.

In Step 3, the principles in Section 4.2.3 are incorporated. In the following, they are introduced as like an immune response process. When a logistical demand is generated, the origin node is chosen by the invasion detection principle. Then, the satisfaction of logistical demand is activated by the invasion response principle. In practical logistics system, the demand may be delayed or cancelled when profit is not ensured. The principle is bio-entity tolerance and channel tolerance. However, in this implementation of the simulation platform, all demands would be handled. Usually, any logistics demand can not be independently handled by a 
node. A series of nodes constitute a route from the origin node to the destination node. The two nodes are given by the logistics demand. This routine of constructing a route is explained by the principle of Idiotype cascading. The above five principles deal with a demand originated from a node. When many demands are satisfied, according to the historical records, the structure of the logistics network would evolve. When an area contains a lot of logistical demands, the nodes within this area would begin to proliferate themselves. The properties of these proliferated nodes would change according to the hypermutation principles. Finally, when the nodes are crowded comparing to the demands, some of them would be closed according to the elimination principle. Therefore, these three principles mainly adjust the logistics network according to the demands and stabilize the logistics network.

\subsection{Experiments}

The simulation procedure in Algorithm 1 is run for 100 times in the experiments. In these experiments, the evolutionary features are focused on, whereas the adaptability and stability are represented by the evolutionary features. In another aspect, adaptability and stability are studied when the structure of the network is relatively fixed. During the process of evolution, the structure, especially the elements of nodes, connections and demands, will be adaptive and tries to be stable. Therefore, the evolutionary tendency analysis also reveals the adaptability and stability.

In the experiments, the parameters are configured as follows.

1) The simulated logistics network is composed of a square grid of $30 \times 50$ road cross points. The road segments have the same length, 1 .

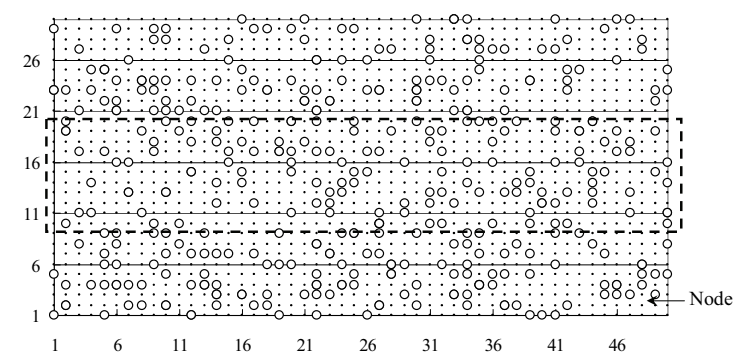

Fig. 1. Simulation grid

2) In order to reveal the tendency of the evolutionary process, a percentage $\operatorname{perc} \in[0,1]$ of the demand nodes are restricted within the area in Fig. 1 where a doted-line frame is surrounded.

3) Logistics demand is satisfied by directly traveling from the demand node to the destination node. Therefore, the energy provided by the demand node is consumed by the origin and target nodes.

4) When the GOTO statement iterates for 1000 times, Algorithm 1 is terminated.

(1) The times of proliferation

In real world, the demands are not distributed equally. In order to simulate this situation, a belt will bigger demands is appointed as shown in Fig. 1. If the demands focus on certain limited areas, the entities will also accumulate around the demands by proliferation and hypermutation. In these experiments, when the demand can not be transported by the nodes within 3 for 5 times, a new node will be generated in this area. In Fig. 2, the mean times of proliferation for each percentage are shown.

It is found that when the demands are increasing focused on the belt in Fig. 1, the proliferation number is decreased. In other words, the structure will be more stable.

(2) The cost evolution tendency

In this experiment, the cost is redefined as the quantities from the demand node to the beginning node. Here the cost is proportional to the distance of the roads that are involved in transport. In Fig. 3, a typical evolutionary process of a population is shown with the decreasing cost.

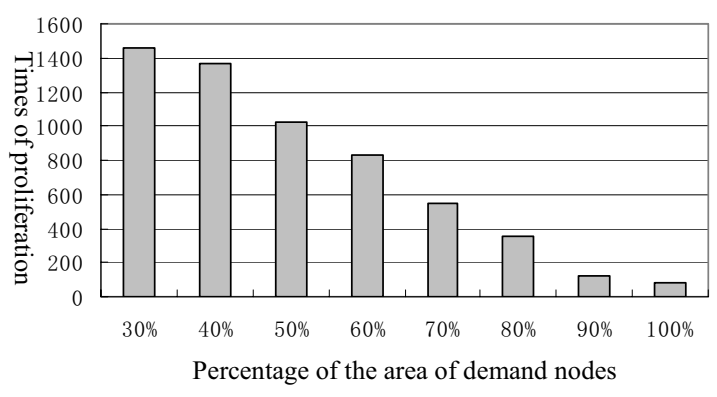

Fig. 2. Proliferation times for different percentages of demand area

In the Fig. 3, each box represents the total cost within 50 times of runs with the same settings. With the process of evolution, the value is decreased to nearly zero. 
Table 2. Maps between bio-network and logistics network.

\begin{tabular}{|c|c|c|}
\hline Eq. & Formulas & Mapped to logistics network \\
\hline$(1)$ & $N E T=(N O D E, C O N N, I N V D)$ & $\begin{array}{l}\text { Logistics network is composed of storage, loading and unloading nodes, transport channels and } \\
\text { logistics tasks. }\end{array}$ \\
\hline (1) & $N O D E=\{1,2, \cdots, N N\}$ & Nodes in logistics network are configured for storage, loading and unloading purposes. \\
\hline (1) & $C O N N=\{1,2, \cdots, N C\}$ & Connections in logistics network are transport channels that connect with nodes. \\
\hline (1) & $I N V D \subseteq N O D E$ & $\begin{array}{l}\text { Storage or transport tasks are demands upon logistics network. Commonly, they are bonded with } \\
\text { specific set of nodes. And some targets are specified. }\end{array}$ \\
\hline (2) & $P N O D E=\left(P_{1}, P_{2}, \cdots\right)$ & $\begin{array}{l}\text { The node properties include node types, storage space and operational capacity. Simply, the type for } \\
\text { different cargos, the storage space, and operation time delay for truck load and cost parameters can be } \\
\text { considered in simple simulation. }\end{array}$ \\
\hline (4) & $P C O N N=\left(P_{1}, P_{2}, \cdots\right)$ & $\begin{array}{l}\text { The properties of connection also basically contain the types, the transport limitations, the traveling } \\
\text { speed and cost. }\end{array}$ \\
\hline (3) & $P N O D E C O N N=\left(P_{1}, P_{2}, \cdots\right)$ & $\begin{array}{l}\text { Commonly, in structural level, the properties on combinations of nodes and connections can be } \\
\text { transferred to be properties of connections. }\end{array}$ \\
\hline (4) & $P I N V D=\left(P_{1}, P_{2}, \cdots\right)$ & $\begin{array}{l}\text { In logistics, the demand can be represented by the cargo storage requirements, storage and transport } \\
\text { means, time window, target nodes and cost requirements. }\end{array}$ \\
\hline (6) & EORI $($ node $)$ & In logistics network, in order to simplify the network, the demand points are attached to the nodes. \\
\hline (6) & $A f f_{n-N}$ & $\begin{array}{l}\text { The affinity between node and the logistics network is defined by the connectivity, the travel time and } \\
\text { distance, and the cost. It can be an aggregation from the affinities between node and node. }\end{array}$ \\
\hline (6) & $A f f_{n-n}$ & $\begin{array}{l}\text { The affinity between node and node in fact is measured by nodes and connections of logistics demands. } \\
\text { The traveling time, distance, and cost are also key factors. }\end{array}$ \\
\hline (7) & $A f f_{c-C}$ & $\begin{array}{l}\text { The affinity between a connection to the connection set is transferred to the affinity between node and } \\
\text { node. }\end{array}$ \\
\hline (7) & $A f f_{c-c}$ & $\begin{array}{l}\text { The affinity between two connections is measured by the affinity between the origin node of the first } \\
\text { connection and the end node of the second connection. }\end{array}$ \\
\hline (7) & $A f f_{i-n}$ & $\begin{array}{l}\text { This affinity deals with matching the logistics demand with a specific node. Commonly, the travel } \\
\text { distance, time, cost and the capacity of node are considered. }\end{array}$ \\
\hline (7) & $A f f_{n-c}$ & $\begin{array}{l}\text { This affinity can be measured by the affinity between the first node and the end node of the second } \\
\text { parameter. }\end{array}$ \\
\hline$(8)$ & INVASION (invd,tw, scale $)$ & $\begin{array}{l}\text { The demand of logistics can be modeled by the demand node, the time-window requirements, and the } \\
\text { involved node set. }\end{array}$ \\
\hline (9) & MATCH & $\begin{array}{l}\text { The travel time, distance, cost and the capacity of the node determines whether it can be chosen to } \\
\text { accept the demand. The historical cooperation records are important. }\end{array}$ \\
\hline (13) & RESPONSE & $\begin{array}{l}\text { The initial network of logistics for the specific demand will basically cover the involved nodes of the } \\
\text { task. Therefore, at least two subsets should be clarified: nodes and connections. }\end{array}$ \\
\hline (15) & TOLERATEN & $\begin{array}{l}\text { It is measured by the capacity matching process between demand and node. The basic capacity includes } \\
\text { storage, time and cost. }\end{array}$ \\
\hline$(18)$ & TOLERATEC & $\begin{array}{l}\text { It can be measured by the capacity matching process between demand and connection. The basic } \\
\text { capacity includes traveling time and cost. }\end{array}$ \\
\hline$(21)$ & $A P P E N D$ & $\begin{array}{l}\text { The efficiency and cost can be employed to determine whether the node is to be appended or removed. } \\
\text { When that the node is append can deduce the cost and improve the efficiency, it should be appended. } \\
\text { However, in practical application, there may be a tradeoff. }\end{array}$ \\
\hline$(22)$ & REMOVE & As discussed in (21). \\
\hline$(25)$ & PDUPLI & $\begin{array}{l}\text { The cost-profit analysis should be the basis for proliferation. In logistics network, this type of } \\
\text { proliferation produces new branches. }\end{array}$ \\
\hline$(28)$ & PSTREN & When the service capacity is strengthened, more resource and risk will be endured. \\
\hline$(30)$ & HYPERM & $\begin{array}{l}\text { New capital will enter the market when there are profit attractions. However, new comer will endure } \\
\text { risks. And, the new comer will break the existing balance. Moreover, it will reorganize the structure of } \\
\text { the network and may optimize the market. }\end{array}$ \\
\hline$(31)$ & ELIMI & $\begin{array}{l}\text { It can be a hidden force of market. When the population scale is beyond the practical requirement, some } \\
\text { nodes will choose to retreat from the field. }\end{array}$ \\
\hline
\end{tabular}


Table 3. Redefinitions of key concepts.

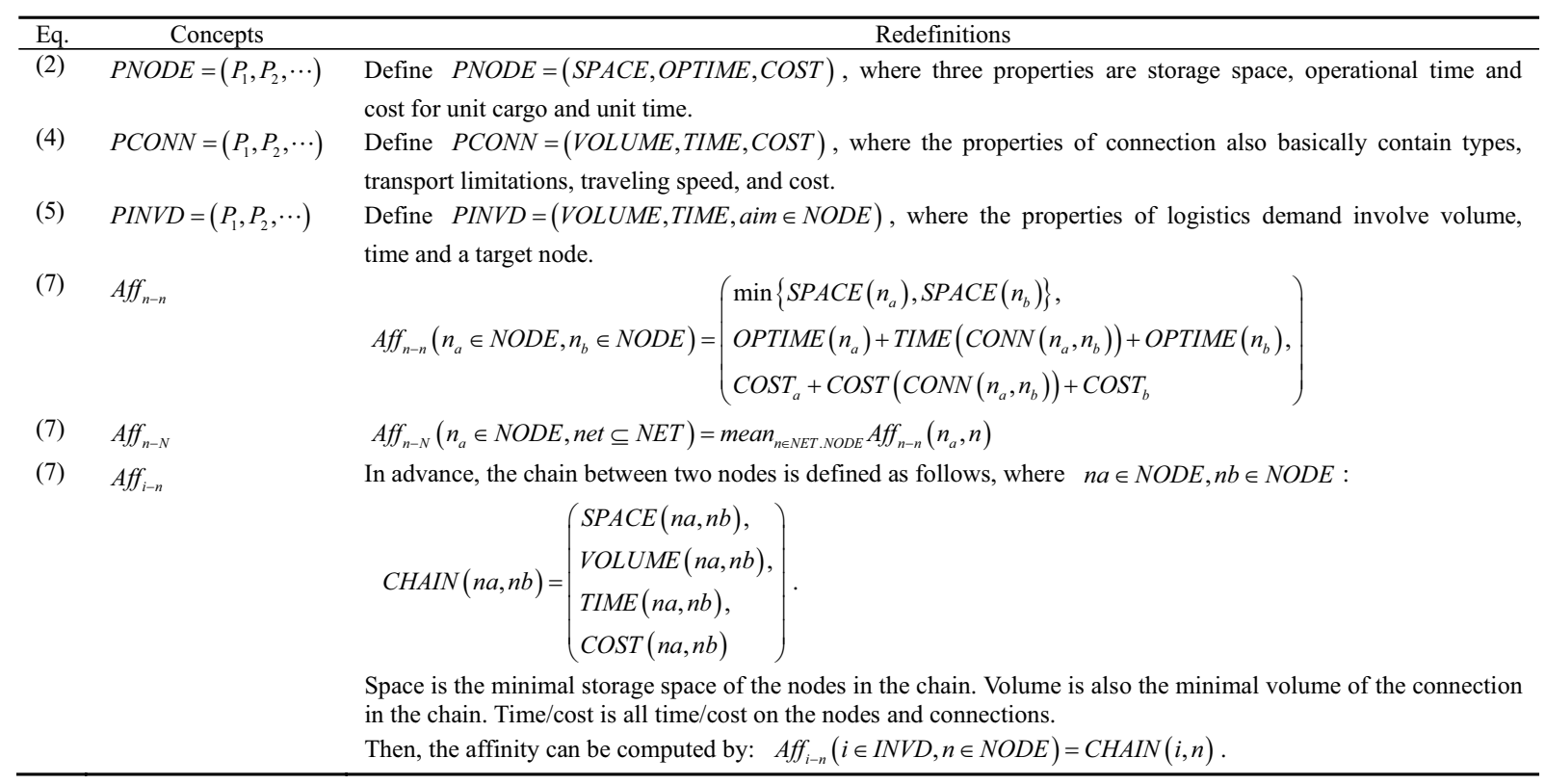

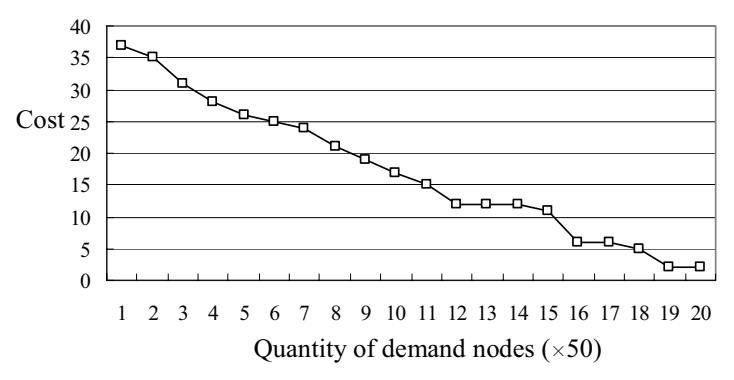

Fig. 3. Evolutionary tendency of cost

\section{Conclusions}

It is beginning to build bio-inspired framework, models and principles for large-scale logistics network. How can the logistics network be modeled in a bio-inspired way? How can the structures and principles inspired by biological systems take actions in assessment, design and optimization tasks for logistics network? This study tries to make a starter answer as generally as possible. The bio-inspired models are studied and eight primary principles are modeled. By simulation, the evolutionary features of logistics network are demonstrated upon the bio-network. However, it is just a beginning. Many simplifications are made in the model and in the simulation study. First, the practical flow and operations should be simulated in the framework, which also should be implemented as a perfect software package. Second, the emergency features of the population are not investigated deeply and fully. Third, the economical environments of logistics network impose great impacts on the evolution and stability of logistics network. A dynamic simulation approach incorporating environmental dynamics will provide a route of revealing the complexity of logistics network as a part of economy. This study only provides the feasibility of studying the evolution and stability features of logistics network when the economical and political impacts are restricted.

\section{Acknowledgments}

Supported by National Nature Science of China (71101088, 71171129, 71390521), China Postdoctoral Science Foundation (2011M500077, 2012T50442) and Science Foundation of Ministry of Education of China and Shanghai (10YJC630087, 20113121120002, 20123121110004, 14YZ100), Science and Technology Commission of Shanghai 
(12ZR1412800, 11510501900, 12510501600), the high-tech research and development program of China (2013A2041106).

\section{References}

1. M.B. Aryanezhad and M. Hemati, A new genetic algorithm for solving nonconvex nonlinear programming problems. Applied Mathematics and Computation, 199(1)(2008) 186-194.

2. S.-T. Bae, H.S. Hwang, G.-S. Cho, and M.-J. Goan, Integrated GA-VRP solver for multi-depot system. Computers \& Industrial Engineering, 53(2)(2007) 233-240

3. Y.-S. Ding, Z.-H. Hu, and H.-B. Sun, An antibody network inspired evolutionary framework for distributed object computing. Information Sciences, 178(24)(2008) 4619-4631.

4. Y. Ding, H. Sun, and K. Hao, A bio-inspired emergent system for intelligent Web service composition and management. Knowledge-Based Systems, 20(5)(2007) 457-465.

5. F. Dressler and O.B. Akan, A survey on bio-inspired networking. Computer Networks, 54(6)(2010) 881-900.

6. V. Furtado, A. Melo, A.L.V. Coelho, R. Menezes, and R. Perrone, A bio-inspired crime simulation model. Decision Support Systems, 48(1)(2009) 282-292.

7. A.T. Gumus, A.F. Guneri, and S. Keles, Supply chain network design using an integrated neuro-fuzzy and MILP approach: A comparative design study. Expert Systems with Applications, 36(10)(2009) 12570-12577.

8. Y. Hamdouch, M. Florian, D.W. Hearn, and S. Lawphongpanich, Congestion pricing for multi-modal transportation systems. Transportation Research Part B: Methodological, 41(3)(2007) 275-291.

9. Z.-H. Hu, Y.-S. Ding, W.-B. Zhang, and Q. Yan, An interactive co-evolutionary CAD system for garment pattern design. Computer-Aided Design, 40(12)(2008) 1094-1104.

10. Z.-H. Hu, A multiobjective immune algorithm based on a multiple-affinity model. European Journal of Operational Research, 202(1)(2010) 60-72.

11. Z.-H. $\mathrm{Hu}, A$ container multimodal transportation scheduling approach based on immune affinity model for emergency relief. Expert Systems with Applications, 38(3)(2010) 2632-2639.

12. Z.-H. $\mathrm{Hu}$ and Y.-S. Ding, An immune inspired co-evolutionary affinity network for prefetching of distributed object. Journal of Parallel and Distributed Computing, 70(2)(2010) 92-100.

13. Z. Hu, Y. Ding, and Q. Shao, Immune co-evolutionary algorithm based partition balancing optimization for tobacco distribution system. Expert Systems with Applications, 36(3, Part 1)(2009) 5248-5255.

14. J.-R. Jung and B.-J. Yum, Artificial neural network based approach for dynamic parameter design. Expert Systems with Applications, 38(1)(2011) 504-510.

15. H.L. Lam, P.S. Varbanov, and J.J. Klemeš, Optimisation of regional energy supply chains utilising renewables:
P-graph approach. Computers \& Chemical Engineering, 34(5)(2010) 782-792.

16. J. Lian, S.M. Shatz, and X. He, Flexible coordinator design for modeling resource sharing in multi-agent systems. Journal of Systems and Software, 82(10)(2009) 1709-1729.

17. L. Michel and P. Van Hentenryck, A simple tabu search for warehouse location. European Journal of Operational Research, 157(3)(2004) 576-591.

18. J.J. Miranda-Bront, I. Méndez-Díaz, and P. Zabala, An integer programming approach for the time-dependent TSP. Electronic Notes in Discrete Mathematics, 36(1)(2010) 351-358.

19. J. Mula, D. Peidro, M. Díaz-Madroñero, and E. Vicens, Mathematical programming models for supply chain production and transport planning. European Journal of Operational Research, 204(3)(2010) 377-390.

20. S. Park and V. Sugumaran, Designing multi-agent systems: a framework and application. Expert Systems with Applications, 28(2)(2005) 259-271.

21. S. Seuring, The product-relationship-matrix as framework for strategic supply chain design based on operations theory. International Journal of Production Economics, 120(1)(2009) 221-232.

22. A. Stauffer, D. Mange, J. Rossier, and F. Vannel, Bio-inspired self-organizing cellular systems. Biosystems, 94(1-2)(2008) 164-169.

23. S. Terzi and S. Cavalieri, Simulation in the supply chain context: a survey. Computers in Industry, 53(1)(2004) 3-16.

24. J. Timmis, A. Hone, T. Stibor, and E. Clark, Theoretical advances in artificial immune systems. Theoretical Computer Science, 403(1)(2008) 11-32.

25. S.M. Wagner and N. Neshat, Assessing the vulnerability of supply chains using graph theory. International Journal of Production Economics, 126(1)(2010) 121-129.

26. Q. Wang, Ideology and Practice of Systems Engineering in Multi-Modal Transport Planning. Journal of Transportation Systems Engineering and Information Technology, 8(1)(2008) 11-16.

27. C. Zhang and C. Zhang, Design and simulation of demand information sharing in a supply chain. Simulation Modelling Practice and Theory, 15(1)(2007) 32-46. 